\title{
doispontos:
}

\section{Individuación, sujeto y aprendizaje ${ }^{1}$}

\author{
Lina Marcela Gil Congote \\ Professora, Universidad de Antioquia, Medellín, Colômbia \\ E-mail: marcela.gil@udea.edu.co
}

\begin{abstract}
Resumen: Este artículo presenta el carácter transductivo de la invención, visto desde el aprendizaje asociado a la individuación psíquica, y a la resolución de problemas asociada a lo colectivo. La discusión se centra en la siguiente tesis: el aprendizaje y la resolución de problemas son invariantes de una psicología de la individuación, desarrollada en cuatro momentos: primero, se recurre a la epistemología simondoniana basada en la analogía y la allagmática para mostrar que no hay individuación sin resolución de problemas; segundo, se desarrolla el continuum que va de las conductas primarias hasta el aprendizaje inteligente y la resolución inventiva de problemas, que encuentra en la técnica una vía paradigmática en Simondon; tercero, se aborda el concepto de sujeto, el aprendizaje y las vías que se abren para lapsicología como disciplina científica y como profesión. Por último, a manera de colofón, se presentan aspectos afines a Simondon en L.Vygotsky y C. Freinet como casos de una perspectiva psicosocial de la resolución de problemas.
\end{abstract}

Palabras clave: Simondon, resolución de problemas, analogía, allagmática, invención, psicología social.

\section{Individuation, Subject and Learning}

\begin{abstract}
This article introduces the transductive nature of invention, from the perspective of learning associated with the psychic individuation, and the problem resolution associated with the collective. The discussion focuses on the following thesis statement: the learning and the problem resolution are invariants of a psychology of individuation, which is developed in four moments: first, simondonian epistemology is used based in the analogy and the alagmatic to show that there is no individuation without problem resolution; second, it will develop the continuum from primary behavior to intelligent learning and problem resolution, which finds in the technique a paradigmatic way in Simondon; third, the concept of subject is approached, the learning, and the ways that are opening to psychology as a scientific discipline and as a profession. Lastly, in the form of a colophon, related aspects to Simondon with $\mathrm{L}$. Vygotsky and C. Freinetwill be presented as cases of a psychosocial perspective in problem resolution.
\end{abstract}

Key-words: Simondon, problem resolution, analogy, allagmatic, invention, social psychology.

\section{Introducción}

Simondon (2016), tomando como referencia la investigación en ciencias humanas en Francia (p.151-173), revisa las tendencias de la psicología en tres direcciones: clínica, experimental y genética; esta última se amplía a genética-pedagógica y considera que es la conciliación de las dos primeras: entre la mirada psicopatológica y psiquiátrica de la enfermedad mental y, la tendencia objetivista de la corriente experimental, basada en estudios anatomo-fisiológicos desde la biología y la medicina (Simondon, 2016, p. 151-154) ${ }^{2}$. Después de un recorrido pormenorizado, que también incluye la psicología social (Dumas, Halbwachs, Blondel), la etnología, la etnografía, la antropología (Durkheim, Lévy-Bruhl, Mauss, Lévi-Strauss), la paleontología 
humana (Teilhard de Chardin), entre otros, concluye que hay una dificultad en las ciencias humanas para regirse por "normas epistemológicas comunes" (Simondon, 2016, p.173), que Simondon esperaba unir en una axiomática común, como lo plantea en Forme, information, potentiels $(2005)^{3}$, en cuya tarea la psicología ocupa un lugar central, capaz de romper la dicotomía entre el psicologismo y el sociologismo, a partir de lo que se identifica como un enfoque psicosocial (Heredia, 2016).

De igual modo, en La résolution de problèmes (2018) cuestiona la existencia de una psicología para estudiar lo individual y otra para estudiar lo social, pero no se ha constituido el paso de una a otra para estudiar la transducción de los procesos psíquicos implicados en el aprendizaje y la invención, mediante el "análisis psicológico de la comunicación” (Simondon, 2018, p. 255). Reconoce que hace falta una psicología de los procesos transindividuales y transductivos (Simondon, 2018, p. 254-255) para estudiar el paso de formas de pensamiento y de la cultura de un sujeto a otro, de una generación a otra en diversas formas: documentos, monumentos, técnicas; en general producciones artísticas, religiosas, literarias, que perduran gracias a la génesis y sucesión de la invención (Simondon, 2018) , $^{4}$ con sus "modalités opératoires et structurales"(Simondon, 2016, p. 324), en la relación indisoluble sujeto-objeto. Sus presupuestos ontológicos y epistemológicos se llevan al terreno de la resolución de problemas para establecer una ley que opera en un germen cristalino, un vegetal, un animal, y en "la genèse même d'images mentales" (Simondon, 2005, p. 189).

El cuestionamiento a la psicología y la valoración que hace del método genético en su vínculo con la pedagogía, viene al caso para enunciar la tesis de este escrito: el aprendizaje y la resolución de problemas son invariantes de una psicología de la individuación. Se desprende de este estudio que el aprendizaje está más asociado a la individuación psíquica, y la resolución de problemas es el enlace con lo colectivo, en su dimensión transindividual, gracias al poder transductivo que caracteriza la invención.

En el primer apartado se recurre a la epistemología simondoniana basada en la analogía y la allagmática para mostrar que no hay individuación sin resolución de problemas. En el segundo apartado se desarrolla el continuum que va de las conductas primarias hasta el aprendizaje inteligente y la resolución inventiva de problemas, que encuentra en la técnica una vía paradigmática en Simondon. Este análisis conduce, en el apartado tres, al sujeto y el aprendizaje y las vías que se abren para la psicología como disciplina científica y como profesión. A manera de colofón, se presentan aspectos afines a Simondon en L. Vygotsky y C. Freinet como casos de una perspectiva psicosocial de la resolución de problemas.

\section{La individuación como resolución de problemas}

En Simondon (2010) el surgimiento de una nueva estructura es como la resolución del problema, cuya información no es reversible (p. 322-325). El como si remite a la analogía sin la cual no puede pensarse la epistemología de la individuación. Las operaciones que acontecen en un ser conocido, en una fase de individuación se llevan, mediante una operación del pensamiento, a un objeto o un problema por esclarecer en otras fases o dominios. Es una transferencia fundada en la "identidad de relaciones operatorias reales" -no en relaciones de identidad de los términos o elementos-. Así, aunque los términos no sean del mismo orden ontológico: una molécula, un cristal, una planta o una imagen mental, el plan de investigación consiste en estudiar los principios de la mecánica cuántica, la física, la cibernética para desentrañar el modo en que operan en otros dominios, esto es, alcanzar un conocimiento válido del esquema genético que es la individuación misma en desenvolvimiento.

Aquí cobra relevancia la allagmática (Simondon, 2015, p. 478-479) ${ }^{5}$, descrita por Simondon en las dos vertientes, como acto: ontogénesis (del orden del ente, óntico) y conocimiento analógico, que requiere 
del análisis y de la analogía (del orden epistemológico, metodológico), a partir de una pregunta metafísica: "quel le est la relation de l'opération et de la structure dans l'être?" (Simondon, 2015, p. 564), considerados estos elementos como "complementos ontológicos" (Simondon, 2015, p. 560)

El orden del ser y del conocer convergen en la transducción. "Elle exprime l'individuation et permet de la penser: c'est donc une notion à la fois métaphysique et logique; elle s'applique à l'ontogenèse et est l'ontogenèse même" (Simondon, 2005, p. 33). El individuo es un sistema transductivo y la transducción es una operación dentro de un sistema; esta no es jerárquica, se conservan los términos iniciales como información. La transducción es el mecanismo mediante el cual se propaga la información amplificando los potenciales disponibles de una región a otra. En el nivel físico la transducción es directa, en el nivel biológico la reproducción de un análogo, gracias a la información genética es transductivo por excelencia; en el nivel psíquico las capas de integración y diferenciación son transductivas, mediante sistemas afectivo-emotivos, perceptivos, conducentes a la acción (Simondon, 2015, p. 234-236); en lo social, normas y valores son transductivas en la medida en que tienen un sentido "optativo", "no clasificatorio" (Simondon, 2015, p. 449), es decir, que se definen en la acción misma dentro de una red de significaciones.

Su programa consiste en un "examen epistemológico" en el dominio físico que se espera sea transductivo en los otros (Simondon, 2015, p. 234-236). Si se logran resolver problemas en otras fases de individuación se puede decir que el conocimiento alcanzado es estable, no solo metaestable, en cuyo caso será sólo un "paradigma elemental" (Simondon, 2015, p. 89-91; 116-118). Advierte que no se trata de una reducción de un "dominio real de lo físico" a "un dominio real de lo viviente" separados por una "frontera real sustancial", sino de una amplificación de estructuras y funciones dentro de un "dominio del conocimiento" de lo físico y lo viviente (Simondon, 2015, p. 412-413; 481-482). Simondon se pregunta si lo físico y lo técnico son el paradigma adecuado para conocer realmente la individuación, dado que su comprensión requiere “(...) el paradigma implícito de la vida que existe en nosotros, que conocemos la operación técnica y la practicamos con nuestro esquema corporal, nuestros hábitos y nuestra memoria" (Simondon, 2015, p. 42; 63).

El psiquismo, la individuación psíquica, está en la base de la epistemología simondoniana porque el sujeto es el ser que conoce y puede comprender la génesis, propia y de los otros seres. Es así como para estudiar la dimensión epistemológica se llega a la psicología y ahora se da el paso al aprendizaje y la resolución de problemas como invariantes de una psicología de la individuación.

El psiquismo es resultado y expresión del desdoblamiento mediante diferenciación de funciones que dotan al ser humano de ciertas estructuras y operaciones a lo largo de la filogénesis y; al mismo tiempo, cada individuo resuelve problemas conforme con su desarrollo, sus potenciales biológicos y las vicisitudes de su trayectoria individual dentro de un entorno que actúa como su complemento. Los problemas surgen del propio individuo, los plantea el entorno o el individuo se toma a sí mismo como problema. Son las variantes que explora Simondon para establecer un continuum desde formas elementales de resolución a formas complejas que incrementan su poder transductivo, como el aprendizaje inteligente y la resoluciónpor invención.

\section{La resolución de problemas en las diferentes fases de individuación}

La individuación es la inserción de un problema en otro, de un nivel prefísico y prevital que se resuelven vía individuación (Simondon, 2015, p. 467), es un "encadenamiento de resoluciones sucesivas" (Simondon, 2015, p. 318), que aumenta en complejidad, siempre con una cuota "residual", un resto que no alcanza a ser individuado (preindividual). En todas las fases de individuación la resolución de problemas desempeña un lugar clave en el despliegue de potenciales. Pero, ¿̇cuáles potenciales se dan en las diferentes fases y qué 


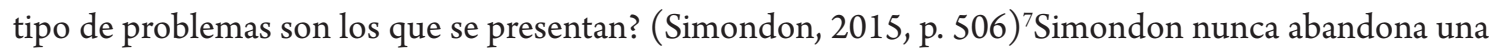
perspectiva psicobiológica para captar la génesis de las conductas desde formas primarias (tropismos y reflejos), secundarias (aprendizajes) y terciarias ("conducta finalizada inteligente con invención y previsión, eventualmente uso de símbolos")(Simondon, 2016, p. 240). ¿Qué es un problema? "Un problème existant 'dès qu'une conduite finalisée rencontre un obstacle à sa réalisation"' (Simondon, 2018, p. 9; Simondon, 2016, p. 467). Con el aprendizaje, la actividad finalizada tiende a desarrollarse cada vez más temprano, a partir de la aparición de estímulos que han tomado el valor de índices o señales.

En grados de complejidad la vida es una constante resolución de problemas, expresados en síntesis, así: 1) Aprehensión o captación de relaciones espaciales, construcción de una topología que inicia en conductas de rodeo o evitación de obstáculos. El mecanismo básico es la sensibilidad como un rudimento de aprendizaje. 2) Adquisición de forma o formalización. La percepción es el mecanismo por excelencia, se da la capacidad de tener un plan y ejecutarlo, mediante el paso gradual de imágenes a símbolos. 3) Esquemas de universalidad que pueden ser transferidos. El caso de la técnica, mediante imaginación e invención, es paradigmático.

En el primer nivel Simondon describe las conductas de rodeo (détour) como la condición mínima de resolución de problemas; afirma que el obstáculo es una forma primera del objeto: aparece una discontinuidad en el entorno para sobrepasar, por tanteo en la forma más primaria o, por ensayo y error, un poco más elaborada. La sensibilidad, tema al que Simondon dedica un estudio completo en Sur la psychologie, es una forma elemental de adaptación. Estudia allí los tropismos: orientación del crecimiento en las plantas por factores lumínicos, químicos, de gravedad, etc; (positivos si crecen hacia el estímulo, negativo si crecen en dirección contraria) y taxismos: movimiento animal por recepción de información a través de nervios (Simondon, 2008, p. 50).

La adaptación implica una diferenciación básica de individuo y medio y mecanismos para sostener funciones nutritivas y reproductivas. Hay resolución elemental de problemas en sistemas rudimentarios de acción como la respuesta a estímulos, salirse de un eje para esquivar un obstáculo (v.g. la lombriz), problemas típicos de rodeo como los laberintos en ratas -o cajas de Thorndike-, en los cuales priman "patrones hereditarios de comportamiento" (Simondon, 2018, p. 74). Estas formas básicas guardan poca relación con el aprendizaje, pero hay fenómenos internos y externos que producen cambios. "La inserción del esquema en el medio exige aprendizajes, muy diferentes de todo juego, e implican un recurso a la información perceptiva, en lugar de la imagen" (Simondon, 2008, p. 50). Se hablará de aprendizaje si en la reorganización del comportamiento, al superar los obstáculos, los ensayos y errores disminuyen (Simondon, 2015, p. 420).

En este nivel no hay propiamente el descubrimiento de un plan general, que es la característica de la resolución de problemas en cuanto tal. Si bien hay condiciones mínimas, no se presenta una "conservación amplificante" propia de las conductas inteligentes, asociadas a la mediación instrumental y a la técnica, donde se conservan y, en algunos casos se transmiten, los modos de producción (Simondon, 2018, p. 92-93).

La percepción -tema central en los estudios simondonianos-asociada a la resolución de problemas, ofrece el tránsito a funciones superiores: "l'activité perceptive établit un continuum stable ayant la fonction d'un système de référence permanent, s'étendant depuis le système sensori-moteur primaire jusqu'aux acquisitions cognitives"(Simondon, 2015, p. 332). Entre lo sensorio-motor y lo cognitivo está la "percepción de tipo superior" que enlaza lo individual (sensorio motor) con "el universo cognitivo colectivo representado por el horizonte espacial y temporal" (Simondon, 2015, p. 333). En este nivel se establecen planes diversos dentro de una organización y un sistema, en el que entran en juego condiciones hereditarias, fenómenos de impresión, condicionamientos y aprendizajes (Simondon, 2015, p. 493). En todos ellos intervienen sensaciones de placer y dolor, así como la identificación de objetos, con sus respectivas valencias. El problema 
que acontece es la "disparidad entre universos perceptivos" y se resuelve con la acción si esta disparidad no es demasiado grande (Simondon, 2015, p. 313).

La acción expresa la interfuncionalidad de los procesos psíquicos: la sensación, forma primaria de adaptación, y la percepción, una forma más elaborada, participan de manera coordinada. La memoria en niveles superiores, cognitivos, no solo retiene o conserva información: representa el pasado (Simondon, 2015, p. 334); pero se diferencia del aprendizaje en que éste, cuando se repite una misma situación, produce o genera una "modificación sistemática" en cierta dirección, y no se queda atada a la información particular del momento (Simondon, 2015, p. 349).

El margen para establecer qué es aprendizaje es muy amplio. Hay aprendizajes por condicionamiento, se insertan aprendizajes a cadenas instintivas. Paralelo al aprendizaje (por impresiones) hay cualidades sensibles que pueden despertar emociones y sentimientos después de lo aprendido, o paralelo con éste, y en cuanto tales son de carácter histórico, individual. Dice Simondon, el aprendizaje -verdadero-:

Es la adquisición de numerosos esquemas (...) integrados que dan al ser humano (...) un poder de plasticidad y de permanente adaptación inventiva. (...) [L]a capacidad de adaptación continuada varía en función directa de la riqueza de la comunicación posible con el medio. Un individuo puede resolver este problema siempre nuevo que es la vida cuando puede comprender y apreciar los resultados de la acción en el medio en el que vive. Cuanto más rápida y precisa sea la información, más eficaz es la autorregulación individual. (...) Ante todo hace falta que la demora de la toma de conciencia de la acción sea corta en relación con la duración de una acción particular (2014, p. 236).

La adaptación inventiva pasa por la comunicación y la acción visible, concreta, que el individuo ejerce sobre su medio. Pero, si la vida es continua resolución de problemas, no siempre es inventiva la respuesta. Cuando se aprende, en primera persona, no necesariamente se transduce en el entorno; en un sentido básico hay modificación de un estado inicial a otro final que puede apreciarse en un nivel básico de conductas de rodeo o asociado a los instintos, pero hablar propiamente de resolución inventiva de problemas trae consigo

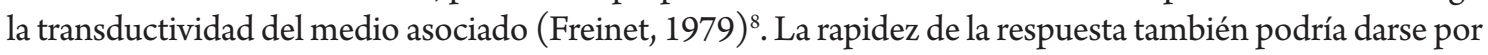
automatismo o entrenamiento, pero la adaptación para ser inventiva requiere la consciencia y la comprensión de la acción generada en el medio y, en consecuencia, saber cómo orientarse eficazmente en situaciones futuras.

La clasificación de Simondon en problemas prácticos y problemas simbólicos (Simondon, 2015, p. 51), ayuda a esclarecer el paso a un nivel terciario. En éste participa un análogo, un modelo, hay algo universal en la resolución de problemas que autoriza la "propagación de un esquema de base" (Simondon, 2018, p. 128). No sólo es diferente lo que pasa en el proceso, también lo que permite la búsqueda, el recorrido en la resolución: una nueva "disposición de objetos", un "gesto" que no estaba antes para el sujeto (Simondon, 2018, p. 131). "El adulto sabe responder a la novedad de las circunstancias del medio a través de la invención inteligente de soluciones: sabe hacer frente a la imprevisibilidad, no por medio de la rigidez de una conducta estereotipada, sino por la riqueza universal de su aprendizaje" (Simondon, 2014, p. 235). Se puede afirmar que el aprendizaje es previo, o es condición necesaria de la resolución de problemas, pero no es suficiente. Se suma la universalidad y la transductividad, como una de las principales características de la invención técnica, aunque no sea exclusiva de ésta.

Se llega así a la resolución de problemas por invención. El aprendizaje se da siempre dentro de las significaciones del grupo, del colectivo. Se pasa de un territorio geográfico -en los animales-a un territorio social (Simondon, 2015, p. 485). Son aprendizajes que activan "connaissances, des motivations, un code de valeurs" (Simondon, 2015, p. 488), y contribuye a la estabilización de actitudes dentro de un medio. Los aprendizajes previos generan estabilidad perceptiva y emocional, que ante situaciones nuevas pueden desestabilizarse y mueven a nuevos ajustes y aprendizajes. 
Uno de los problemas que enfrenta la individuación psíquica y colectiva es no perder su potencial mediante significaciones que se renueven y en efecto devengan en invención, bien sea técnica, lingüística, ética y política (Bardin, 2015). Solo en la medida en que participa de la individuación de lo colectivo, el sujeto resuelve "la disparidad de su problemática" (Simondon, 2005, p. 244).

El aprendizaje inteligente es una forma de llamar a la resolución de problemas en un nivel más complejo que se logra mediante la invención. En La individuación a la luz de las nociones de forma y de información y $D u$ mode d'existence des objets techniques se refiere a "Fases sucesivas de un proceso de génesis" que desarrolla en Imagination et Invention bajo el ciclo de la imagen, pasando por el símbolo y la concretización de objetos técnicos. Una de las características de la invención es rebasar el fin previo, se da un "salto, un poder de realización" (Simondon, 2008, p. 196), que no se limita al problema previsto; por su carácter aleatorio surgen "propiedades nuevas" y las soluciones pueden llevarse a otros ámbitos, con extensión más duradera (Simondon, 2018, p. 107-109)9 mientras que la creatividad suele ser más instantánea (Simondon, 2018, p. 207). Es también la diferencia con la mediación instrumental presente en los animales (Simondon, 2018, p. 91) ${ }^{10}$.

La invención trasciende la inmediatez de la solución (como puede pasar en la creatividad); la invención no es individual, ni la creatividad es meramente un efecto de grupo, es una sucesión de fases y conductas en "relación efectiva de interacción" (Simondon, 2018, p. 153-154) ${ }^{11}$. El pensamiento religioso, ético, social y político, técnico, más concretamente la tecnicidad, porta consigo esquemas cognitivos y axiológicos que se actualizan en la resolución de problemas que ha dado lugar a la concretización del objeto técnico y su inserción en la cultura. El encadenamiento en el tiempo y el progreso de las técnicas otorgan un "poder de expansión y universalidad de empleo", que es fuente para la psicología a partir del estudio de la génesis en las fases previas.

La resolución de problemas por invención rebasa "las condiciones del problema en potencia" y se universalizan las soluciones (Simondon, 2008, p. 199). El objeto técnico es para Simondon producto del aprendizaje inteligente, es "símbolo concreto que permite la relación interhumana más rica y universal" (Simondon, 2014, p. 237), "cristaliza en una estructura funcional el dinamismo de un esfuerzo de conciencia y de acción” (Simondon, 2014, p. 237).

La acción mediante la técnica se inserta en la cultura como resolución de problemas que valen para uno y valen para todos. Cultura y técnica, con sus respectivos contenidos mentales y axiológicos, son fuentes de educación; pero tiende a darse un dualismo que no solo separa, sino que reserva su enseñanza para ciclos diferentes del desarrollo. Para Simondon convendría invertir el orden: más temprano la tecnicidad, la cultura en la edad adulta (Simondon, 2014, p. 337). Por supuesto son simultáneos en el orden de la experiencia, pero no en la comprensión del funcionamiento o en los potenciales que permite desarrollar. Entre ellos, quizá el más importante, el pensamiento reflexivo para ir a la génesis de los procesos y tomar consciencia de los múltiples fines que se desprenden de la acción.

\section{El sujeto y el aprendizaje}

Dentro de un sistema tenso en el que siempre hay nuevos problemas por resolver, el sujeto psíquico es resultado y agente del problema dentro de un haz de relaciones: se pone problemas a sí mismo, participa de la solución dentro de sistemas cognitivos y axiológicos. Pero los niveles de los problemas varían y se abren vertientes diferentes para una psicología de la individuación.

El aprendizaje por su propia naturaleza de esquemas que se ponen en funcionamiento lo vive cada quien, no puede darse sin la participación de un sujeto concreto en la situación. Pero, no se llega a la 
universalización si las soluciones no son transferibles o quedan abiertas para que otros puedan hacer uso de ellas o acoplarse en situaciones similares. Los efectos del aprendizaje en rigor no son individuales, la necesidad de comunicación pone de manifiesto su carácter transindividual, el cual se consolida cuando se traduce en esquemas universales a partir de la invención.

Viene al caso la distinción de Piaget entre sujeto psicológico: se plantea problemas del ámbito individual que se adquieren mediante aprendizaje. El sujeto epistémico remite a esquemas universales y se plantea problemas que valen para uno y valen para todos. Este último es sujeto de conocimiento, "un sujeto cualquiera portador de los mismos dispositivos que poseen todos los sujetos individuales del mismo nivel de desarrollo" (Battro, 1971, p. 352-353). La epistemología genética estudia, dentro de un nivel evolutivo y del desarrollo, los aspectos comunes de los sujetos sin tener en cuenta las diferencias individuales. El sujeto epistémico ejerce una función evaluativa, establece la generalidad de los problemas planteados, o se pregunta si éstos son del ámbito de la ignorancia subjetiva. El sujeto psicológico es una vivencia que en Piaget se descentra para ocuparse del conocimiento válido y su incremento (Simondon, 2016, p. 323-324; Gil y Vargas, 2019) ${ }^{12}$.

Este descentramiento de la experiencia subjetiva, del yo empírico o del sujeto psicológico, es solo una de las vertientes posibles. A la psicología también le interesa el nivel de resolución de un sujeto en su trayectoria cuando estudia los procesos de aprendizaje en entornos específicos con sujetos, grupos y colectivos. Desde un modelo general epistemológico se llega a explicar las estructuras y operaciones de la individuación de cualquier ente, incluyendo el psíquico, en términos teóricos, si se quiere eidéticos. Pero, al mismo tiempo, el sujeto se individua psicológica o empíricamente: viviendo, individualizándose. Para Simondon: "El ser individuado es el sujeto trascendental y el ser individualizado el sujeto empírico" (Simondon, 2005, p. 391). Los problemas son del ámbito trascendental, y las respuestas o el conjunto de soluciones son del sujeto empírico, una experiencia individualizada, un sujeto con una personalidad (síntesis de ambos) y un carácter o trayectoria individual.

Un sujeto que se formula problemas a sí mismo, se involucra en la solución; aquel que conoce y puede desentrañar una génesis. La misma que opera en las diferentes fases de individuación y puede conocerse por analogía: identidad de relaciones que develan la "condición constitutiva, energética y estructural, que se prolonga en la existencia de los seres constituidos" (Simondon, 2005, p. 83).

Se puede decir: el sujeto epistémico es transhistórico, no está personalizado; mientras que el aprendizaje que vive el sujeto obedece a su marco de experiencia, a sus disposiciones biológicas y las contingencias de su trayectoria individual; este aprendizaje alcanza el ámbito de la resolución de problemas a medida que logra mayor transductividad y se inserta en una red de problemas, dentro de un horizonte común de acción (Simondon, 2015) ${ }^{13}$.

La resolución no es propiedad de un individuo, sino de los sistemas creadores de forma. Desde la herencia de Leroi- Gourhan y Bergson, en diálogo con Bateson (Ingold, 2010, p. 6-25), la formación acontece en un espacio, es una relación de movimientos y objetos, que abarca, cuerpos, ambientes, ritmos, movimientos y acciones en sus flujos. El aprendizaje es un proceso inmanente de relaciones del sistema del cual se es parte; es una integración metaestable de funciones, tanto cognitivas como afectivo-emotivas; éstas últimas son "un principio de constancia entre lo cognitivo y lo operatorio, una fuerza que hace posible cualquier función de relevo [relé], y por lo tanto mantiene un nivel medio de vigilancia y acción, que podría llamarse 'homeostasis del comportamiento" (Simondon, 2018, p. 55); es también un "enlace regulador entre percepción y acción" (Simondon, 2018, p. 13). Así visto, el aprendizaje y la resolución de problemas nunca cesa, es un campo relacional que controla un flujo de información de diferentes órdenes, en contexto, situado, dentro de una individuación más amplia que la del propio individuo (Sautchuk, 2015, p. 21). 
El aprendizaje es condición necesaria de la resolución de problemas. El primero hace parte de los procesos que dan lugar a la individuación psíquica; el segundo es el modo en que lo aprendido se amplifica y consolida lo colectivo; ambos conforman la dimensión transindividual.

\section{Colofón: enfoque psicosocial del aprendizaje y la resolución de problemas}

Desde diversas tradiciones se puede establecer un diálogo con Simondon para entender el carácter psicosocial de los procesos psíquicos. Se tomarán a modo de cierre dos autores de vertientes diferentes dentro de la misma época: L.S. Vygotsky (1896-1934) y C. Freinet (1896-1966). Al menos cuatro aspectos guardan resonancia con la obra de Simondon y desde ellos se podrá avanzar en las bases conceptuales de una psicología de individuación: 1) Sistema individuo-entorno; 2) Interfuncionalidad de los procesos psíquicos; 3) Despliegue de potenciales en un entorno que transforma y es transformado; 4) Perspectiva político-pedagógica visible en su manera de pensar el sujeto y el devenir desde la resolución de problemas.

1. Vygotsky sostiene que el paso de las funciones inferiores o elementales a las superiores, no es una suma de elementos, sino una "síntesis" de nuevas combinaciones en el desarrollo sociocultural del comportamiento, que conjuga maduración y aprendizaje. Surgen leyes diferentes mediante la superación de las anteriores. En el sentido hegeliano advierte que superar tiene la doble connotación de suprimir, negar, pero al mismo tiempo conservar. Como una paradoja "cada estadio superior niega al inferior, pero lo niega sin destruirlo, sino incluyéndolo como categoría superada, como un momento integrante" (Vygotsky, 2012, p. 119).

Más que un tronco común del que se desprenden ramas (procesos psíquicos), hay un ascenso en términos de complejidad (apoyado en leyes neurobiológicas) que se expresa en la síntesis de procesos superiores independientes. Hay un sistema jerárquico que se organiza a partir del pensamiento, de la formación de conceptos que reorganiza, reestructura todos los procesos básicos y superiores: Sensación (sensibilidad), percepción, atención, memoria, participan en el aprendizaje así como del pensamiento, el lenguaje, la inteligencia y la consciencia, en cada nivel evolutivo y del desarrollo.

En el caso de la edad de transición, de la infancia a la adolescencia, estudiada por el autor, la memoria pasa de ser mecánica a compleja, esto es, lógica, vinculada a conceptos. En el caso de la percepción cada estadio cambia cualitativamente, del bebé al niño, de éste al adolescente. Es lo que denomina "edad de transición" en la que se pasa de una síntesis primaria a una secundaria. La constancia del objeto (magnitud, forma, color, etc.) se alcanza de manera gradual y con el desarrollo de la memoria, la percepción se une al pensamiento y al lenguaje (Vygotsky, 2013) ${ }^{14}$. En el paso de lo elemental a lo superior la percepción no es la suma de las partes, es un proceso en el que el lenguaje orienta la atención, modifica la percepción, la descompone y sintetiza con juicios verbales, "se intelectualiza la percepción", lo aislado se regula y "adquiere sentido, pasado y futuro" (Vygotsky, 2012, p. 123). Del pensamiento concreto de los niños, con significaciones afectivas, se pasa en el adolescente a los conceptos y el pensamiento abstracto, que no se limita a tomar conciencia de lo percibido. En el primer estadio del desarrollo los objetos se determinan por su "función efectiva", pero el lenguaje, la formación de conceptos, permite pasar propiamente a un objeto de conocimiento mediante un pensamiento racional.

Las operaciones empiezan con medios externos y se van incorporado en el comportamiento mediante lo que denomina "ley de arraigo" (Vygotsky, 2012, p. 144) en un vínculo indisoluble entre desarrollo (interno) y aprendizaje (externo). El primero es condición necesaria para el segundo, pero se requiere integración, interiorización de signos e instrumentos del lenguaje. No basta la maduración o los procesos evolutivos porque la presencia de los otros es necesaria y en la relación con ellos el sujeto se transforma. 
Entiende la conducta desde la plasticidad de las funciones neuropsicológicas y las condiciones del entorno. Al estudiar las mediaciones (regular conductas con "recursos metacognitivos, por ejemplo) no se puede ver el sujeto como pasivo o reactivo, un objeto de experimentación desligado de sus componentes sociohistóricos: más allá de los test y la homogeneización, es necesario conocer al niño y a quienes conviven con él. Signos, útiles, pautas de los otros (pares, adultos, maestros) conforman la zona de desarrollo próximo o potencial: lo que el niño o joven puede hacer con ayuda de otros.

2. Como caso paradigmático de un enfoque psicosocial en el aprendizaje se encuentra Célestin Freinet en la ruptura de la escuela tradicional y la apertura a la Educación nueva, valorada en Simondon desde un método genético-pedagógico. Si alguien lleva con sus matices el asunto del aprendizaje social es Freinet. El mundo escolar es parte integrante del mundo social y dispositivos como la prensa escolar, el aprendizaje y la lectura hacen de enlace entre esos dos mundos (Vargas, 2006, p. 247-255).

La clase opera "como medio técnico de vida" e incorpora en su enseñanza -para dar el giro hacia el aprendizaje-, la "clase paseo", la salida del aula al campo, para favorecer la participación y, en efecto, transformar el medio y transformarse con él. La comunicación es el motor del aprendizaje y el "medio técnico que hace posible la comunicación es la imprenta, el diario y la correspondencia interescolar" (Simondon, 2015, p. 266-267).

Las necesidades concretas de la vida, no los ambientes o las pautas artificiales dirigen el aprendizaje: el texto y la expresión oral libres, el "cálculo vivo" en la medición y los intercambios cotidianos con la comunidad, mueven una constante "voluntad de comunicación". La imprenta genera espontáneamente la corrección de la lengua, la ortografía, la puntuación, la amplificación de la comunicación con lo más próximo y a gran escala, con la comunidad y con el mundo, dejando a un lado la "gramática inútil" o la repetición sin sentido de lo que se lee. Dice Freinet:

Escrutaremos, pues, el alma del niño para lo cual disponemos de una técnica que ha demostrado ser lo suficientemente operativa: el texto libre, la imprenta escolar y la correspondencia interescolar. Esta expresión espontánea será a la vez un desarrollo de las personalidades y ocasión escolar para adquirir, amplificar y precisar las diversas adquisiciones: lengua, gramática, vocabulario, ciencias, historia, geografía, moral, introduciendo lógicamente, en el interés infantil así exteriorizado, disciplinas previstas en el programa (1979, p. 200).

Para Simondon, en Freinet la imprenta escolar es un "medio polivalente" de "composición del texto, imaginado, escrito. Es un método para desarrollar habilidades manuales, memoria visual, lectura, escritura y ortografía, dentro de la jornada de la escuela. En centros de menores infractores la edición de un boletín, juega un rol psicosocial importante: otorga a los delincuentes, "que se sienten aislados de la sociedad, un medio de expresión hacia el exterior", estar en la imprenta, interactuar con ella es un test, más apropiado que los aplicados de manera artificial para evaluar al niño infractor dentro de un "interrogatorio judicial", incluso vergonzoso para el niño o el joven (Simondon, 2016, p. 266-267).

Su método convoca la resolución de problemas, mediante la técnica artesanal, la fabricación de objetos para la interacción con los otros, la creación artística libre (pintura en edades más avanzadas) y la invención colectiva de medios de comunicación; en todos ellos se reconoce la expresión libre, incluso inconsciente de potencias anímicas, de motivos que se disponen al encuentro con otros. La afecto-emotividad, vía la resolución de problemas en el ámbito transindividual, es también moduladora de la experiencia para que acontezca lo transindividual.

Contra la escolástica y frente al poder establecido, oficial, visible también en los textos, el "poder es transferido a los propios alumnos". El aprendizaje responde a una necesidad y la escuela es un medio vital, un lugar de encuentro comunitario, que pone en vilo el afán de posesión y poder del capital (Legrand, 
1993). El aula cooperativa y los proyectos conjuntos sustentan su orientación política y pedagógica en resonancia con el poder transductivo del aprendizaje y la toma de conciencia que favorece la individuación psíquica y colectiva, transindividual. Como dice Legrand: "Freinet quería introducir la vida en la escuela en una época en que la escuela era un templo 'de paredes desnudas” (1993, p. 12).

Construye una teoría y un método del aprendizaje escolar mediante los siguientes principios: "a. Permeabilidad a la experiencia (...). b. Huella dejada en el comportamiento (...).c. Tanteo experimental (...). d. Intuición (...). e. Autonomía (...). f. Acto logrado (...). g. Gradación del aprendizaje” (Vargas, 2006, p. 249).

Estos principios se traducen en una incorporación progresiva de modos de hacer también en un continuum que pasa por niveles más sensoriales hasta la racionalización de la experiencia y los efectos de "autorresponsabilidad" y "valoración crítica" (Vargas, 2006, 253). Los motivos del niño conducen a experiencias significativas que se explicitan cada vez más de manera racionalizada por medio de tanteos experimentales que reinician el ciclo a nuevos potenciales de tanteo y aprendizajes; en términos simondonianos se vinculan esquemas cognitivos y esquemas axiológicos que transforman al sujeto y éste, de retorno, transforma el medio en su constante interacción. Las diferentes experiencias y los contenidos aprendidos pueden ser comparados y llevados a contextos diferentes, mediante el uso de la técnica y la comunicación. Desde una ontología del presente: ofrece "una estrategia metódica para hacer una recepción pedagógica de los dispositivos tecnológicos, al tiempo que una recepción tecnológica de los dispositivos pedagógicos" (Vargas, 2006, p. 255).

Freinet propone una teoría escolar del aprendizaje que se puede leer simondonianamente para expresar su vigencia en los entornos de aprendizaje y de resolución inventiva de problemas, en especial en el ámbito de la democracia, la participación y la formación ciudadana, que toma en nuestro país el camino de la formación para la paz.

Tanto Simondon, Vygotsky y Freinet ofrecen tesis fundantes de un enfoque psicosocial que, llevado al aprendizaje y la resolución de problemas, perfila una psicología de la individuación tanto en su vertiente teórica y epistémica, como en su dimensión práctica, en el acompañamiento de sujetos en entornos concretos. No obstante, es importante diferenciar una teoría general del aprendizaje, al modo en que Vygotsky sentó las bases del método genético de los procesos psíquicos superiores, de una teoría del aprendizaje escolar donde el maestro aporta en el diseño de ambientes, como es el caso de Freinet. En ambos el aprendizaje se da en el ámbito de la experiencia, de las condiciones que puede o no propiciar el entorno para desarrollar potenciales. Se puede afirmar que Vygotsky aporta a la dimensión teórica de la psicología en la comprensión de las estructuras y los procesos psíquicos en su interfuncionalidad y propone formas de llevar a la práctica, en condiciones normales y de déficit, la pedagogía basada en un enfoque socio-histórico, socio-cultural. La propuesta de Freinet enfatiza el saber dentro y fuera del aula como una experiencia psicosocial, sin llegar necesariamente a generar una teoría universal del funcionamiento de las estructuras y las operaciones que participan en el aprendizaje y la resolución de problemas. Ambos enfoques se emparentan en el método: genético en Vygotsky, genético-pedagógico en Freinet. Su complementariedad dentro de una psicología de la individuación, en sintonía con principios simondonianos, está por desarrollarse. En cualquier caso, no se puede perder de vista que la psicología cuenta históricamente con diversas vertientes: clínica, experimental y genética (Simondon, 2016, p. 153-154), y el modo como se combinen depende del interés del investigador, el tipo de problemas que se formule y el método -o los métodos- que adopte. Si el eje de estudio es la individuación tendrá que ocuparse del proceso transductivo entre lo individual y lo social, bien para conocer las operaciones y estructuras del sistema individuo-entorno (una de ellas el aprendizaje), bien para acompañar y ayudar el devenir de la resolución de problemas. 


\section{NOTAS}

1. Texto apresentado durante o COLÓQUIO INTERNACIONAL GILBERT SIMONDON: OS SENTIDOS DA INDIVIDUAÇÃO, realizado no Departamento de Filosofia da Universidade de São Paulo, de 05 a 07 de dezembro de 2018 (nota do editor). O artigo é derivado da Pesquisa "Psicología de la individuación en entornos formativos para la paz. Individuo, sistema y diferencia” (Cod. 2018-23425) (nota da autora).

2. En la primera ubica el enfoque psicopatológico de la psiquiatría, el psicoanálisis (Lagache, Anzieu, Lacan), el psicodrama y otros estudios sobre la enfermedad mental y la psiquiatría general (Ey, Guiraud, Minkowski). En la segunda ubica tendencias renovadas del mecanicismo para el estudio de las sensaciones, la percepción, los reflejos. Se destaca Fraisse en la propuesta de integrar sistemas diferentes de explicación, más que un método exclusivo. No obstante, priman la medición, el control, el cálculo, las matemáticas, la teoría de juegos y demás recursos para la experimentación en psicología, con "objetividad metodológica", acompañada de propuestas pedagógicas y ciclos propedéuticos para su enseñanza en facultades de Letras y Ciencias Humanas. Se da el auge de aplicaciones en el campo del trabajo, el aprendizaje, consejería, psicología escolar. La tercera se caracteriza por estudios sobre la psicología del niño (Le Senne, Berger, Mesnard), con tendencia a una filosofía de la educación pero con "observación directa”, capaz de articular método antiguos y nuevos (Chateau). Se abren centros de formación para psicólogos y educación especial. Destaca Wallon, quien representa una vertiente objetivista con el estudio de factores sociales y personalidad, pedagogía y vida colectiva, que conducen al movimiento francés de escala mundial Educación nueva (Éducationnouvelle), entre los que se encuentran el trabajo libre por grupos (Cousinet), la cooperación escolar (Profit), el autogobierno (Demolins), cercanos al movimiento del colectivo infantil de Makarenko y Monoszon, al igual que Freinet con la imprenta escolar. Piaget por su parte introduce la epistemología genética, el estudio de estructuras que organizan la experiencia de un estadio al otro; más que elemento y totalidad refiere vínculos, lazos (Liason) dentro de un "estructuralismo operacionalista" (pp. 152-158).

3. "Quisiéramos mostrar que un esbozo de axiomática de las ciencias humanas o al menos de la psicología es posible si se intenta captar en conjunto las tres nociones de forma, información y potencial, a condición de añadir, para enlazarlas y organizarlas interiormente, la definición de un tipo particular de operación, que aparece cuando hay forma, información y potencial: la OPERACIÓN TRANSDUCTIVA" (Simondon, 2015, p. 481-482). No obstante, pone en duda esta posibilidad: "il reste à découvrir une axiomatique de l'ontogenèse, si toutefois cette axiomatique est définissable. Il se peut que l'ontogenèse ne soit pas axiomatisable, ce qui expliquerait l'existence de la pensée philosophique comme perpétuellement marginale par rapport à toutes les autres études, la pensée philosophique étant celle qui est mue par la recherche implicite ou explicite de l’ontogenèse en tous les ordres de réalité» (Simondon, 2005, p. 229).

4. Génesis y sucesión de la invención, incluso no técnica, como es el caso de la historia de las fases que ha seguido la sociedad en sus formas de gobierno.

5. La allagmática combina una "ciencia analítica": descompone el todo en sus partes, estudia los elementos o su combinación para conocer la estructura; y, una "ciencia analógica” que toma la totalidad para estudiar las equivalencias entre operaciones.

6. Las "intuiciones" de las que parte Simondon son tomadas del modelo de la química y la física para el caso de la cristalización y para el caso de la modulación del modelo de la información. Sin embargo, no desarrolla la hipótesis de la allagmática en extenso para integrar ambos modelos y llegar a la causalidad y la noción del devenir en las diferentes fases (Simondon, 2015, p. 479-480). Posterior a este texto, el concepto de información le servirá para llegar a la transducción y poner en segundo plano el concepto de allagmática. Aunque el concepto no aparezca con frecuencia en su obra y el de información -al igual que transducción- se conserva de manera transversal, quizá por ser más fecundo, la descripción del vínculo con la analogía es de utilidad para comprender el modo en que concibe el ser en su devenir.

7. Esta teoría se distinguiría del innatismo realista (ligado a la teoría arquetípica) y del empirismo nominalista (ligado a una teoría hilemórfica): el progreso del conocimiento sería efectivamente una formalización, pero no un empobrecimiento ni un alejamiento progresivo que abandone lo concreto sensorial; la formalización sería una 
adquisición de forma, consecutiva a una resolución de problema: señalaría el pasaje de un estado metaestable a un estado estable del contenido de la representación.

8. Corresponde al tanteo experimental que conduce a una experiencia lograda.

9. En La résolution de problèmes (2018) propone la invención como un proceso intelectual por etapas: sincrética, analítica, sintética. Análisis y síntesis son complementarios, propone el concepto de transferencia para el paso de una a otra, que luego introduce como transducción. En el caso de invenciones no técnicas, por ejemplo en la filosofía, ubica estas mismas fases.

10. En los animales hay mediaciones instrumentales complejas, i.e. vertebrados superiores se sirven de armas y herramientas, pero se diferencia de los humanos en el tiempo consagrado a la herramienta, antes y después del uso, y su prolongación en la cadena de actos: preparar, conservar, reparar.

11. Elabora así su crítica en Résolution de problèmes (2018) al método de Osborne y en general a los cursos y manuales para favorecer la creatividad, mediante técnicas como la lluvia de ideas. La creatividad no reemplaza la invención, en la que se pone en juego una estructura, una solución universalizable.

12. El conocimiento válido es terreno de la epistemología, que abarca en Simondon los diferentes modos de pensamiento con menor o mayor grado de estabilidad. Así como el sujeto de conocimiento es al mismo tiempo afecto-emotividad (motivación) y, acción u operación (voluntad), el objeto y la relación con éste se da tanto a nivel cognitivo y de las representaciones que alcanzan el estatuto de ciencia, como a través de fundamentos culturales: tecnología, estética, praxeología.

13. El aprendizaje en Simondon no es aislado, acontece dentro de las significaciones de un grupo que activa conocimientos, motivaciones y valores (Simondon, 2015, p. 485); el aprendizaje se expresa en actitudes que llegan a estabilizarse en el medio (p. 489). Si las normas y los valores no se conectan con los actos de otros, en la cadena de actos, en las significaciones que se construyen con otros, pierden su poder de acción: "L'acte est ce qui dans la vie est susceptible d'être réfléchi, soit avant, soit après l'accomplissement; nous ne voulons pas dire par là que l'acte est toujours le fruit d'une décision préalable; mais il est ce qui est racine de réflexion, et s'insère entre une réflexion antérieure et une réflexion postérieure; l'acte se situe d'emblée sur un terrain réflexif comme résultat d'une différenciation et point de départ d'une intégration; or, ce qui est intégration à l'intérieur du sujet est différenciation à l'extérieur, et réciproquement” (Simondon, 2015, p. 444).

14. Los estudios sobre descripción de dibujos en los niños muestra las siguientes etapas: 1) Enumerar objetos representados -3 años-; 2) describir acciones; 3) indicios de representación; 4) Descripción de relaciones entre las partes, correlaciones en un todo coherente. De manera breve se siguen las fases de: objeto, acción, cualidad y relación.

\section{REFERÊNCIAS}

BARDIN, A. Epistemology and political philosophy in Gilbert Simondon: Individuation, Technics, Social Systems. Londres: Springer, 2015.

BATTRO, A. M. Diccionario de epistemologíagenética. Trad.FlorealMazia. Buenos Aires: Proteo, 1971.

CHEHONADSKIH, M. The Communist Drama of Individuation in Lev Vygotsky. In: Stasis, Vol. 5, n. 2, 2017, pp. 110-135.

FREINET, C. Los métodos naturales. Barcelona: Editorial Fontanella, 1979.

GIL, L.; VARGAS, G. “The Psychology of Individuation as Epistemology”. In: PhilosophyToday: Special Issue on Simondon (en prensa). 
HEREDIA, J. “Lo psicosocial y lo transindividual en G. Simondon”. In: Revista Mexicana de Sociología, vol. 77, n. 3, 2015, pp. 437-465.

INGOLD T. “Da transmissão de representações à educação da atenção”.InEducação, vol. 33, n. 1, 2010, pp. 6-25.

LEGRAND, L. "Célestin Freinet, un creador comprometido al servicio de la escuela popular” (LR). Publicado originalmente en Perspectivas: Revista trimestral de educación comparada (París, UNESCO: Oficina Internacional de Educación), vol. XXIII, n. 1-2, 1993, pp. 425-441.

PIAGET, J. Biología y conocimiento. Trad. Francisco González Aramburu. México: Siglo XXI, 1969.

SAUTCHUK, C. E. “Aprendizagem como gênese: prática, skill e individuação”. In: Horizontes antropológicos, vol. 21, n. 44, 2015, pp. 109-139.

SIMONDON, G. Du mode d'existence des objets techniques.Paris: Aubier, 1989.

. L'individuation à la lumière des notions de forme et d'information. París: PUF, 2005.

. (2005). L'invention dans les techniques. Cours et conférences. Paris: Seuil, 2005.

. "Allagmatique" dans L'individuation à la lumière des notions de forme et d'information. Grenoble: Éditions Jérôme Million, 2005.

. "Forme, information, potentiels" dans L'individuation à la lumière des notions de forme et d'information. Grenoble: Éditions Jérôme Million, 2005.

. "Note complémentaire sur les conséquences de la notion d'individuation" dans L'individuation à la lumière

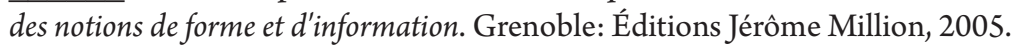

. Cours sur la Perception (1964-1965). Paris: Éditions de La Transparence, 2006.

. Imagination et Invention (1965-1966). Paris: Éditions de La Transparence, 2008.

. Communication et information. Cours et conférences. Paris: Les Éditions de La Transparence, 2010.

. Sur la technique. Paris: PUF, 2014.

. "Allagmatica". In: La individuación a la luz de las nociones de forma y de información. Buenos Aires: Cactus, 2015, pp. 469-480.

Sur la psychologie. Paris: PUF, 2015.

. "Nota complementaria sobre las consecuencias de la noción de individuación”. In: La individuación a la luz de las nociones de forma y de información,2015, pp. 435-468. [Segunda edición corregida y aumentada]. Buenos Aires: Cactus, 2015b.

. "Forma, información y potenciales". En: La individuación a la luz de las nociones de forma e información. [Segunda edición corregida y aumentada]. Buenos Aires: Cactus, 2015, pp. 481-511.

Sur la Philosophie. Paris: PUF, 2016.

La résolution de problèmes. Paris: PUF, 2018. 
VYGOTSKY, L. “Desarrollo de las funciones psíquicas superiores en la edad de transición”. In: Obras escogidas. Tomo IV. Madrid: Machado, 2012.

. "Historia del desarrollo de las funciones psíquicas superiores". InObras escogidas. Tomo III. Madrid: Machado, 2012.

“Pensamiento y lenguaje”. In: Obras escogidas. Tomo II. Madrid: Machado, 2014.

Paidología del adolescente. In: Obras escogidas. Tomo IV. Madrid: Machado, 2012.

VARGAS, G. Filosofía, pedagogía, tecnología. Bogotá: San Pablo, 2006.

VIRNO, P. Cuando el verbo se hace carne: lenguaje y naturaleza humana. Trad. Eduardo Sadier. Madrid: Traficantes de Sueños, 2005. 